\title{
Ward Round - Paediatric bowel obstruction: A surprising and rare cause of a common problem
}

\section{Jonathan C. Samuel ${ }^{1,2}$, Arturo P. Muyco ${ }^{3}$}

1. NC Jaycee Burn Center, Department of Surgery, University of North Carolina, Chapel Hill, NC, 27599, United States.

2. UNC Project, Private Bag A-104, Lilongwe, Malawi

3. Department of Surgery, Kamuzu Central Hospital, PO Box 149, Lilongwe, Malawi.

Corresponding author: Dr. Jonathan Samuel, NC Jaycee Burn Center, Department of Surgery, University of North Carolina, Chapel Hill, NC, 27599, United States.

\section{Description of the case}

A four year old boy presented to Kamuzu Central Hospital for evaluation of five days of progressive nausea, vomiting, and obstipation. History was obtained from the child and mother. For several months prior to presentation he suffered from abdominal discomfort and distension, without diarrhoea, constipation, fevers, chills, or weight loss.

On physical examination, he was found to be normothermic, mildly tachycardic at 120 beats per minute, and normotensive. Respiratory rate and oxygen saturation were within normal limits. He appeared well nourished, appropriately developed both physically and mentally. Chest examination was unremarkable. On abdominal examination, he had significant distension with visible peristaltic waves, tympanic to percussion, and diffuse abdominal tenderness without guarding or rebound. There appeared to be a mass in the left mid-abdomen. On digital rectal examination he had nonbloody stool with normal rectal tone. Enemas failed to relieve his symptoms. He was given intravenous fluids and antibiotics, and a nasogastric tube was inserted.

Further workup entailed laboratory and radiographic studies and a surgical consultation. Full blood count showed a mild leukocytosis and a haematocrit of 42. An HIV test was performed and was negative. Ultrasound of the abdomen was performed and revealed what appeared to be a multiloculated cystic mass arising from the anterior abdomen. Erect and supine radiographs showed a large round structure in the left mid-abdomen with an air fluid level. The child was taken to theatre for exploration, with a provisional diagnosis of mechanical obstruction due to either a tumour or intussusception.
Figure 1 Specimen showing multiple subserosal cystic lesions filled with air and fluid, with one dominant lesion and associated small bowel obstruction.

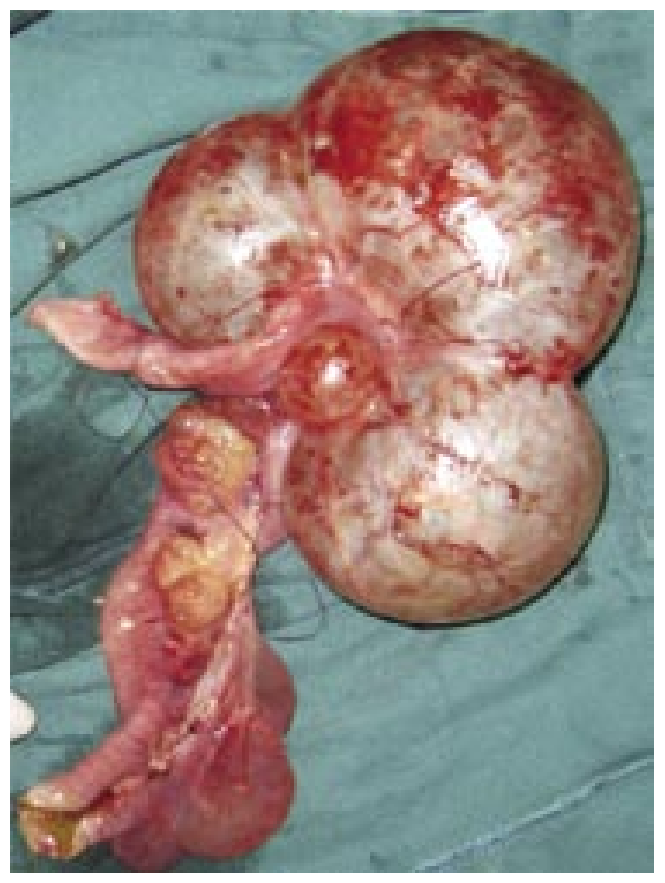

Operative findings revealed a $15 \mathrm{~cm}$ segment of jejunum with multiple cystic lesions. The remainder of the abdomen was normal. The proximal intestine was significantly dilated proximal to the largest of the cystic lesions, with the distal small bowel decompressed, consistent with complete small bowel obstruction. All of the cystic lesions, including the dominant lesion at the apparent transition point, were filled with a combination of gas and clear yellow fluid. The mesentery and vasculature were normal, and the entirety of the small bowel, aside from the obstruction, appeared well perfused. The affected segment of small bowel was resected, and primary end-to-end enteroenterostomy was performed (figure 1).

What caused the bowel obstruction in this child? Continued on page 102 


\section{Ward Round - Paediatric bowel obstruction: A surprising and rare cause of a common problem}

\section{Continued from page 100}

\section{Discussion of the case}

Initial evaluation and management of bowel obstructions follows a standard approach. The patient should be supported with hydration and bowel rest, including gastric decompression if emesis is persistent. Vital signs and urine output should be closely monitored to assess intravascular volume status. Examination should focus on findings that can suggest aetiology, such as presence of abdominal scars to suggest adhesions, inguinal hernia causing mechanical obstruction, abdominal masses, or peritoneal signs such as guarding or rebound tenderness.

Radiographic evaluation often includes upright and supine radiographs, which should be reviewed for the presence of air fluid levels, dilated loops of bowel, gas in the rectum, or pneumoperitoneum. Pneumoperitoneum, or free air within the abdomen, can be seen under the right diaphragm on upright abdominal films, and necessitates emergent surgical evaluation and almost always operative intervention. Other signs of significant obstruction, such as dilated loops and air fluid levels, suggest significant pathology and should be managed with serial abdominal examinations and surgical evaluation. CT scan is often helpful in the evaluation of bowel obstruction, however this is not available at our institution.

In neonates, the aetiology of bowel obstruction includes intestinal atresias and stenoses, malrotation, meconium ileus, Hirschsprung's disease, peritoneal bands, and congenital hernias. Infants with obstruction often suffer from pyloric stenosis, intussusception, duplication cysts, and hernias. Appendicitis, adhesions from prior surgery, hernias, duplication cysts, tumours, and malrotation predominate as causes in children over 24 months of age ${ }^{1}$.

Akgur et al retrospectively evaluated 228 cases of adhesive bowel obstruction in children, and found four criteria useful in predicting vascular compromise: abdominal pain, tachycardia, leukocytosis, and fever'. When three or all four criteria are present, $50 \%$ had vascular compromise at the time of operation. This child presented with abdominal tenderness, leukocytosis, and tachycardia, which in light of the evidence suggests a high probability of vascular compromise, and hence an indication for surgery.

Gross pathology revealed Pneumatosis Cystoides Intestinalis (figure 1), which is defined by gas-filled cysts within the bowel wall. The cysts are located either in the submucosal or subserosal layers of the intestinal wall ${ }^{3}$. In this case, histopathology was not available. Pneumatosis Cystoides Intestinalis is considered a subset of Pneumatosis Intestinalis, which generally refers to conditions of gas within the bowel wall, cystic or otherwise 4 .

Pneumatosis Cystoides Intestinalis is a rare condition. There are several case reports in adults ${ }^{5,6}$, and even fewer in the paediatric age group 7 . The largest series in nonneonatal paediatric patients, reported from the University of Colorado, included 32 patients over an 8 year period 8 . Of these children, $22 \%$ were healthy, while the remaining had chronic diagnoses, the most common of which included congenital heart disease, motility disorders, gastroschisis, and short bowel syndrome. The most common preceding events prior to presentation were non-infectious colitis $(32 \%)$, acute enteric infection (27\%), bowel ischemia (20\%), and gastrointestinal dysmotility (17\%).

Management of Pneumatosis Cystoides Intestinalis is usually conservative. Surgery is reserved for cases of intestinal perforation, necrosis, or complete bowel obstruction. Emphasis should be on finding one of the aforementioned underlying causes of Pneumatosis Cystoides Intestinalis, though some cases, such as this one, are idiopathic.

\section{References}

1. Derbew, M. Neonatal Surgical Emergencies. In: Ostrow, B ed. Surgery in Africa Monthly Review, Ptolemy Project, University of Toronto; Dec 2005.

2. Akgur FM, Tanyel FC, Buyukpamukcu N, Hicsonmez A. Adhesive small-bowel obstruction in children: predictors of vascular compromise of the intestine. Pediatric Surgery International 1992 Mar; 7(2):113115

3. D'Agostino S, Fabbro MA, Musi L, Bozzola L. Pneumatosis Cystoides Intestinalis: A Rare Cause of Nonsurgical Pneumoperitoneum in an Infant. Journal of Pediatric Surgery. 2000 Jul; 35(7):1106-1108.

4. St. Peter SD, Abbas MA, Kelly KA. The Spectrum of Pneumatosis Intestinalis. Archives of Surgery 2003 Jan;138(1):68-75

5. Aziz MR, Wandwi WB, Mkony CA. Pneumatosis cystoides intestinalis-in Tanzania. Central African Journal of Medicine. 1995 Jul; 41(7):230-233.

6. Ibara BI, Bossali F, Ossendza RA, Ollandzobo LC, Ibara JR, ItouaNgaporo A. Pneumatosis cystoides intestinalis: the first case in Congo [article in French]. Sante. 2001 Apr-Jun; 11(2):131-132.

7. Fahal AH, Baraka OZ, el-Lider AM. Pneumatosis cystoids intestinalis: a case report. East African Medical Journal. 1994 Jun; 71(6):401-402

8. Kurbegov AC, Sondheimer JM. Pneumatosis Intestinalis in Nonneonatal Pediatric Patients. Pediatrics. 2001 Aug; 108(2):402-406 